\title{
Medicating patients during extracorporeal membrane oxygenation: the evidence is building
}

\author{
Amy L. Dzierba1*, Darryl Abrams ${ }^{2}$ and Daniel Brodie ${ }^{2}$
}

\section{Abstract \\ This article is one of ten reviews selected from the Annual Update in Intensive Care and Emergency Medicine 2017. Other selected articles can be found online at http://ccforum.com/series/annualupdate2017. Further information about the Annual Update in Intensive Care and Emergency Medicine is available from http://www.springer.com/series/8901.}

\section{Background}

Extracorporeal membrane oxygenation (ECMO), which can support gas exchange or hemodynamics in patients with severe respiratory or cardiac failure, has demonstrated considerable evolution over the last decade [1], with a steady rise since 2009 in the number of ECMOtreated patients and number of centers providing ECMO support $[2,3]$. With more adult patients being placed on ECMO support, there is an increased need to understand the complex changes in drug pharmacokinetics and pharmacodynamics that occur with the addition of an ECMO circuit to the management of a critically ill patient.

The relationship between the dose of a drug and the elicited response may be altered in critically ill patients as a result of pharmacokinetic and pharmacodynamic changes [4]. The use of extracorporeal mechanical support, such as ECMO, can further increase the variability of pharmacokinetic alterations [5]. Therefore, the combination of critical illness and ECMO presents considerable challenges to providing optimal pharmacotherapy. The ability to anticipate alterations in pharmacokinetics and pharmacodynamics in this patient population is essential

\footnotetext{
* Correspondence: ald9012@nyp.org

${ }^{1}$ Columbia University Medical Center, Department of Pharmacy, NewYork-Presbyterian Hospital, New York, NY 10032, USA Full list of author information is available at the end of the article
}

for providing an individualized therapeutic plan that maximizes therapeutic benefit while minimizing potential toxicity.

Despite improvements in extracorporeal technology and resurgence in its use in respiratory and cardiac failure, there remains a paucity of data on pharmacotherapy in patients receiving ECMO. This chapter summarizes our current understanding of the effects of ECMO on the pharmacokinetics and pharmacodynamics of several drug classes commonly used to manage these critically ill patients.

\section{The effect of critical illness and ECMO on pharmacokinetics}

Pharmacokinetics encompasses the absorption, distribution, metabolism and elimination of a drug, ultimately influencing the concentration at the targeted site of action. In contrast, pharmacodynamics denotes the relationship between the drug concentration and the physiologic and biochemical effects of the drug on the body, including the intensity of therapeutic and adverse effects. The relationship between pharmacokinetics and pharmacodynamics is influenced by the drug, the underlying disease process, and the presence of extracorporeal factors. The culmination of all of these factors can lead to considerable and unpredictable alterations in pharmacokinetics. Figure 1 summarizes the effects of serum drug concentrations and the resultant pharmacokinetic changes influenced by critical illness and ECMO.

The elimination of drugs from the body is highly dependent on clearance of the drugs from the body and on volume of distribution. The patient's physiology and the specific physiochemical properties of drugs, such as protein binding, hydrophilicity, molecular weight, and degree of ionization at a given physiologic $\mathrm{pH}$, may influence both clearance and volume of distribution. The presence of ECMO frequently leads to additional alterations, including an 


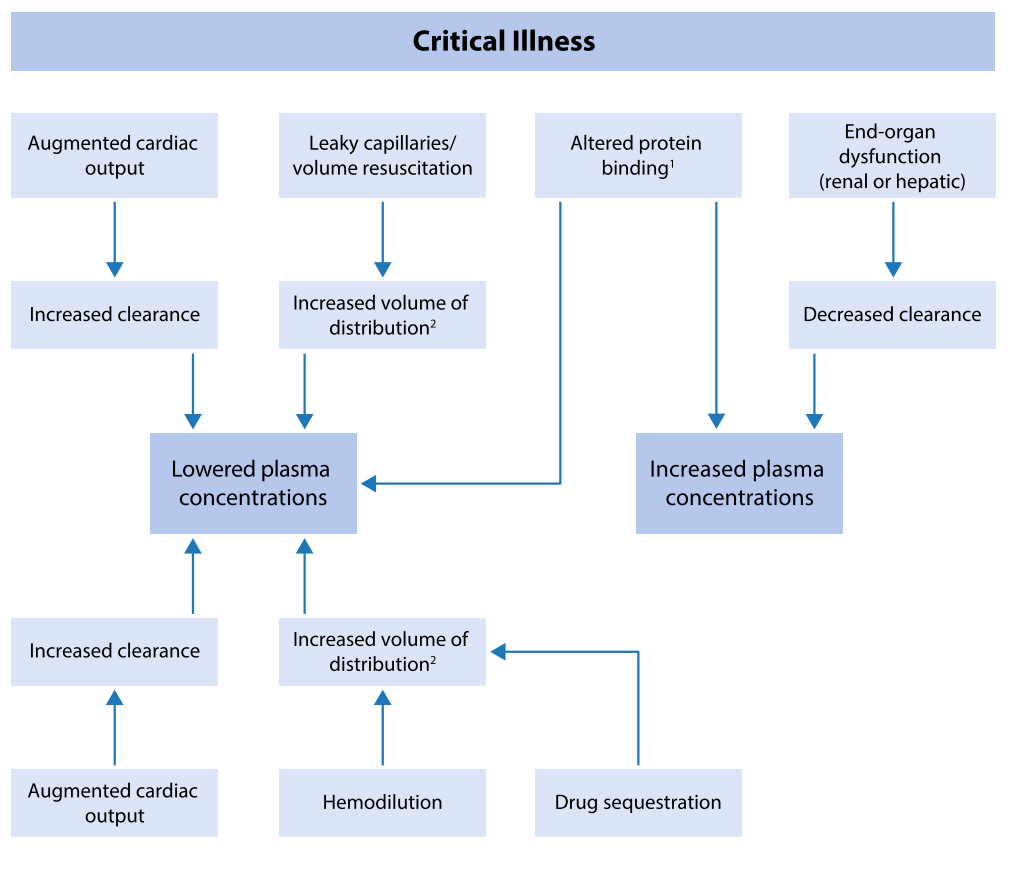

Extracorporeal Membrane Oxygenation

Fig. 1 Changes in pharmacokinetics with critical illness and extracorporeal membrane oxygenation. ${ }^{1}$ increased a1-acid glycoprotein and decreased albumin concentrations; ${ }^{2}$ mostly affecting hydrophilic drugs

increased volume of distribution and either increased or decreased drug clearance [5].

The liver and the kidneys are the two major organ systems responsible for drug metabolism and elimination, with less significant elimination occurring through the biliary system, gastrointestinal tract and lungs. Critically ill patients often develop organ insufficiency or failure during the course of their illness thereby altering drug elimination rates. Decreased renal blood flow or function will lead to a decreased glomerular filtration rate, affecting drugs that are dependent on this route of elimination (mainly hydrophilic drugs), whereas decreases in hepatic perfusion or function may lead to toxicity through decreased enzymatic activity or decreases in extraction efficiency.

The distribution of drug throughout the body (volume of distribution) is largely dependent on the drug's hydrophilicity and its acid-ionization constant. Drugs that are hydrophilic will have a lower volume of distribution and their concentrations will primarily be influenced by fluid-shifts and large-volume fluid resuscitation. In contrast, lipophilic drugs penetrate into the tissues, leaving lower concentrations in the blood and increasing the apparent volume of distribution. The ECMO circuit may increase the volume of distribution through either hemodilution or sequestration of drugs [5, 6]. An initial increase in volume of distribution that occurs at the initiation of ECMO from the introduction of priming solutions (plasma, saline, or albumin), primarily affecting hydrophilic drugs, may result in decreased plasma concentrations and, potentially, therapeutic failure of a drug. This increased volume may also lead to the dilution of plasma proteins, notably albumin, affecting drugs that are highly protein-bound, leading to potential toxicities as a result of an increase in the proportion of the unbound fraction of a drug.

Modern extracorporeal circuits typically consist of cannulae, polyvinyl chloride (PVC) tubing, a centrifugal pump, and an artificial membrane for gas exchange (often termed a 'membrane oxygenator'). The membrane oxygenator and PVC tubing comprise a large surface area for potential drug sequestration, which may lead to drug loss over time, particularly for lipophilic drugs $[5,7,8]$. The composition of the tubing and membrane oxygenator may play a role in the amount of drug that is sequestered. Some studies have observed that both PVC tubing and the membrane oxygenators absorb drugs to a similar extent, whereas others have shown significant differences. This may be explained by both the age of the circuit and the type of pump used [8-12].

Albumin and $\alpha_{1}$-acid glycoprotein, both synthesized in the liver, are the two major blood proteins that bind to drugs. In critical illness, albumin concentrations decrease 
in response to increased vascular permeability, decreased production and increased catabolism; whereas, $\alpha 1$-acid glycoprotein is an acute-phase reactant that may increase in response to physiologic stress. The changes in protein concentrations could affect both the amount of free drug available and the overall volume of distribution. Additionally the deposition of protein on the inner surface of the ECMO tubing may potentially increase the sequestration of drugs that have a high affinity for protein binding [13]. A recent ex vivo model tested the changes in concentrations over time of highly protein bound drugs [14]. Highly protein bound drugs in this experiment included ceftriaxone, caspofungin and thiopentone, all of which had significantly lower mean drug recoveries at $24 \mathrm{~h}(80,56$, and $12 \%$, respectively) compared to drugs that were not highly protein bound [14].

Since lipophilic and highly protein bound drugs are more prone to sequestration in ECMO circuits, an understanding of the physicochemical properties of drugs can assist in determining the relationship between the dose administered and the anticipated blood concentration [5, 15]. The octanol-water partition coefficient or $\log P$, is a common way to report the measure of a drug's lipophilicity [16]. Drugs with high $\log P$ values (around 2.0) will have a propensity to be very soluble in organic materials such as the PVC tubing used in the ECMO circuit. However, to date, there has been no characterization of the drugcircuit interaction beyond $24 \mathrm{~h}$ and, as such, little is known regarding the adsorptive capacity of the circuit over longer periods of ECMO support. Table 1 summarizes the effects of critical illness and ECMO on pharmacokinetics of drugs based on degree of lipophilicity of the drug.

\section{Analgesia and sedation}

Patients receiving ECMO often require analgesia and sedation to reduce oxygen consumption, facilitate patientventilator synchrony, diminish patient stress and discomfort, and prevent patient-initiated device dislodgement or

Table 1 Effect of critical illness and extracorporeal membrane oxygenation (ECMO) on pharmacokinetics (PK) of drugs, based on degree of lipophilicity

\begin{tabular}{lll}
\hline & Hydrophilic drugs & Lipophilic drugs \\
\hline $\begin{array}{l}\text { Volume of distribution }(\mathrm{Vd}) \\
\begin{array}{l}\text { Primary mode of } \\
\text { clearance }\end{array}\end{array}$ & Low & High \\
Log $P$ & Hepatic \\
$\begin{array}{l}\text { Potential effect of critical } \\
\text { illness on PK }\end{array}$ & Increased Vd & High \\
Effect of ECMO on PK & Increased Vd & Increased Vd \\
& No change in clearance & Increased clearance
\end{tabular}

Log P: octanol-water partition coefficient (measure of drug's lipophilicity) removal [1]. However, achieving the desired level of sedation and preventing delirium in the critically ill patient receiving ECMO remains a challenge in ICUs, owing to paradigm shifts in analgosedation regimens as well as pharmacokinetic alterations of commonly used analgesics and sedatives. Medication selection should be based on the patient's needs, with titration to a predetermined goal in accordance with recently published guidelines [17]. Limited data exist on the most appropriate opioid and sedative medication regimen to use in ECMO patients to achieve the desired level of sedation while minimizing excess sedative exposure. Commonly used intravenous opioids include fentanyl, hydromorphone and morphine, while sedatives often include propofol, dexmedetomidine and benzodiazepines. The choice of agent used is dependent on the desired physiological endpoint while balancing unwanted adverse effects of each individual agent.

Three ex vivo studies using adult ECMO circuits composed of PVC tubing and a hollow polymethylpentene fiber membrane oxygenator demonstrated significant losses of commonly used opioids and sedatives $[6,18,19]$. One investigation observed as much as a $93 \%$ loss in dexmedetomidine concentrations at $24 \mathrm{~h}$ [18]. Another study, measuring concentrations of morphine, fentanyl, and midazolam over a 24-hour period, demonstrated that the average drug recovery relative to baseline was lower with lipophilic drugs [6]. Only $3 \%$ of fentanyl and $13 \%$ of midazolam were detectable at $24 \mathrm{~h}$; however, the ECMO circuit did not substantially alter morphine concentrations, with $103 \%$ recovery at $24 \mathrm{~h}$. Of interest, in the first hour of ECMO support, up to 70 and $50 \%$ of fentanyl and midazolam, respectively, were lost in the circuit [6]. The most recent ex vivo study using adult ECMO circuits primed with human whole blood observed a recovery of only $30 \%$ of baseline propofol concentrations at $30 \mathrm{~min}$ and negligible concentrations at $24 \mathrm{~h}$ [19]. Additionally, midazolam concentrations were substantially reduced with 54 and $11 \%$ of baseline concentrations measured at $30 \mathrm{~min}$ and $24 \mathrm{~h}$, respectively [19]. Similar findings have also been observed in neonatal circuits composed of PVC tubing and earlier generation silicone membrane oxygenators. These investigations observed up to a $68 \%$ loss of midazolam and a 98\% loss of propofol within $40-120 \mathrm{~min}$ and up to a $30 \%$ reduction in lorazepam concentrations $[11,12]$.

Adult patients receiving ECMO for respiratory failure appear to have increased requirements of analgesia and sedation over time [20,21]. The first case report demonstrating increased sedation requirements was in a 30year-old man with severe respiratory failure requiring venovenous ECMO as a bridge to lung transplantation. Over 19 days of ECMO support, his requirements of morphine and propofol to maintain deep levels of 
sedation increased significantly [20]. A small, single-center, retrospective study observed an increase in opioid and sedative requirements over time in 29 consecutive patients requiring ECMO (13 venovenous, 16 venoarterial) where the sedation protocol was to keep patients heavily sedated during the first few days of ECMO followed by daily lightening of sedation when possible [21]. This study reported an increase in the average daily dose of midazolam and morphine of $18 \mathrm{mg}(p=0.001)$ and $29 \mathrm{mg}(p=0.02)$, respectively [21]. Interestingly, the authors did not find any significant increase in fentanyl requirements over time. Additionally, patients receiving venovenous ECMO had a significantly higher daily midazolam dose requirement than did patients receiving venoarterial ECMO $(p=0.005)$ [21]. A more recent single-center, prospective cohort study set out to determine the median daily dose of opioids and benzodiazepines in 32 patients receiving either venovenous or venoarterial ECMO [22]. In this mixed cohort the median daily dose of opioids and benzodiazepines, 3875 micrograms and $24 \mathrm{mg}$ respectively, was found to be lower than that reported from previous trials. Additionally, the authors did not find increasing requirements throughout the duration of ECMO support. The lower opioid and benzodiazepine requirements observed in this study could be a result of the study institution's analgosedation approach, lower goal sedation scores, and use of other non-benzodiazepine sedatives [22].

The only comparative trial to date is a recent retrospective cohort study that enrolled consecutive adult patients with severe respiratory failure with $(n=34)$ or without $(n=60)$ venovenous ECMO support requiring at least one sedative to maintain a level of wakefulness appropriate to maintain patient comfort and safety while optimizing oxygenation and ventilator support [23]. The authors found that the maximum median 6-hour sedative exposure was nearly twice as high in the ECMO group and was reached nearly 3 days later when compared to the group not receiving ECMO. However, there was no significant difference in 6-hour sedative exposure in adjusted analyses [23]. Therefore, this study challenges whether the increased requirements of opioids and sedatives are a result of circuit-related factors alone or whether other factors, such as tolerance, age or pharmacogenomics, play a central role.

Existing data are sparse to guide the appropriate dosing of opioids and sedatives in adult patients receiving ECMO in the context of modern extracorporeal technology. Many of the first-line agents used in the management of pain and sedation are lipophilic and therefore have a high propensity to be adsorbed or sequestered by the extracorporeal circuit $[6,11,12]$. One approach to achieving adequate sedation in patients receiving ECMO would be to start with continuous infusions of both an opioid and a sedative, anticipating requirements that exceed standard doses with the ultimate goal of minimizing the deleterious effects of sedative agents, especially benzodiazepines, with daily interruptions or down titrations. Additionally, clinicians should anticipate the need for significant dose reductions at the time of ECMO discontinuation given the likely rapid decrease in volume of distribution. Failure to do so could result in overuse of these medications. The reduction in dosing may be difficult to calibrate, so it is prudent to carefully monitor for signs of withdrawal and delirium. Consideration of adjunct agents, such as sub-anesthetic doses of ketamine, may help facilitate achieving sedation goals. Two uncontrolled studies demonstrated reductions in sedative rates with the addition of low-dose ketamine infusions $[24,25]$. Most recently, a randomized trial did not show any differences in opioid or sedative requirements with the addition of low-dose ketamine to standard sedation practices as compared to standard sedation practices alone in patients receiving venovenous ECMO for severe respiratory failure [26]. Standard sedation practices consisted of infusions of fentanyl or hydromorphone and midazolam to achieve a Richmond Agitation Sedation Scale (RASS) of -5 at the initiation of ECMO. The median cumulative amount of fentanyl and midazolam equivalents in the low-dose ketamine group were almost twice and four times as high, respectively, when compared to the control group from ECMO initiation to the decision to achieve wakefulness [26]. However, patients receiving low-dose ketamine infusion had similar improvements in their RASS scores over the 72hours after the decision to achieve wakefulness [26].

The incidence of delirium in patients receiving ECMO is not well characterized; however, given the high use of benzodiazepines in these patients, it may be reasonable to presume that the rates are as high as those reported in the critically ill patient population not receiving ECMO [27]. When appropriate, the use of a regimented analgosedation approach, daily interruption of sedation, and early mobilization may help minimize opioid and sedative exposure and thus reduce the incidence of delirium associated with these drugs, as appears to be the case in critically ill patients in general [17].

\section{Antimicrobials}

Infections are commonly encountered in critically ill patients and are associated with higher mortality [28]. In a critically ill patient, source control, in addition to timely and appropriate antimicrobial administration, remains the cornerstone of successful treatment of infection [29]. Selecting the appropriate dose of an antimicrobial can be challenging given the potential effects of critical illness and ECMO on drug concentrations, particularly considering that most antimicrobial dosing 
regimens have been established in healthy adults with normal physiology [5]. Changes in volume of distribution and clearance from critical illness and the ECMO circuit may affect pharmacodynamic parameters that ultimately determine the effectiveness of the antimicrobial agent. Inappropriate antimicrobial dosing may result in substantial drug losses, leading to therapeutic failure, development of resistance, and worse outcomes in patients with life-threating infections whereas an empiric increase in dose may potentially lead to accumulation and toxicity.

Vancomycin, a moderately protein bound, hydrophilic antimicrobial agent is commonly used to treat Grampositive bacterial infections. Two in vitro ECMO studies observed steady vancomycin drug concentrations over 24 and 48-hour periods [6, 19]. In a matched cohort study of adult critically ill patients, those receiving ECMO had a similar volume of distribution and clearance of vancomycin compared to those not receiving ECMO in the first $24 \mathrm{~h}$ of therapy [30]. All patients received a $35 \mathrm{mg} / \mathrm{kg}$ loading dose over $4 \mathrm{~h}$, followed by a continuous infusion targeting a serum concentration of 20-30 mg/l [30]. Linezolid, an alternative Gram-positive antimicrobial, was studied in three adult patients receiving ECMO, the results of which suggest that therapeutic targets may not be achieved with standard dosing when the minimum inhibitory concentration (MIC) is greater than $1 \mathrm{mg} / \mathrm{l}[31]$.

Aminoglycosides, including gentamicin and tobramycin, are hydrophilic drugs with low protein binding and with increased volume of distribution in the context of critical illness, resulting in decreased maximal concentrations. Additionally, in the context of a higher volume of distribution from the ECMO circuit, standard or higher initial doses may be needed with normal or extended intervals in order to provide sufficient peak concentrations. The study of aminoglycoside pharmacokinetic alterations during ECMO is largely limited to the neonatal population; however, one observational study in adult patients demonstrated comparable pharmacokinetics with amikacin in critically ill patients with or without ECMO support [32]. Therapeutic drug monitoring is readily available for this class of antimicrobial agents, making it feasible to target effective concentrations while limiting potential toxicity.

Extended-spectrum penicillins, cephalosporins and carbapenems are commonly used in the treatment of Gram-negative infections in the critically ill patient population. As a class, these antimicrobials are generally hydrophilic, largely dependent on renal elimination, and have moderate to low protein binding; however, variability exists with certain drugs such as ceftriaxone. Optimizing the time-dependent, bactericidal effect of this class will be achieved by maximizing the time concentrations above the MIC. The use of extended or continuous infusions seems to be a reasonable approach to optimize the pharmacodynamics in critically ill patients receiving ECMO [4].

Conflicting data have been reported on meropenem, a hydrophilic carbapenem with low protein binding. While some studies suggest a significant loss of meropenem within the ECMO circuit [6,33,34], other investigations have found no effect in vivo compared to other critically ill patients [35]. A recent retrospective, case-control study observed no differences in pharmacokinetic paramenters with either piperacillin/tazobactam or meropenem in patients receiving ECMO compared to those not receiving ECMO [35]. Of interest, nearly $30 \%$ of all drug levels measured for the two aformentioned drugs were subtherapeutic, which may be a consequence of other pathophysiological disturbances not controlled for in this critically ill population [35]. Mechanical circulatory support in general can induce a systemic inflammatory response, independent of the underlying critical illness, which, in turn, may augment renal clearance. Classes of antimicrobials in which direct correlations of augmented renal clearance and lower serum drug concentrations have been observed include beta-lactams, aminoglycocides and glycopeptides [36].

Regarding other antimicrobial drug classes, azithromycin pharmacokinetics appear to be similar between patients receiving ECMO and non-ECMO critically ill controls [37] and tigecycline levels in one patient were similar to expected levels based on population pharmacokinetics [38]. Concentrations of ciprofloxicin, a fluoroquinolone that is lipophilic with low to moderate protein binding, do not seem to be affected by ECMO [14]. To date there are no data on pharmacokinetic changes with polymyxin B or polymyxin E (colistin) that are used to treat multi-drug resistant Gram-negative infections. ECMO does not appear to affect oseltamivir pharmacokinetics directly; however, patients with renal dysfunction may experience impaired drug clearance [39-41].

Data on the pharmacokinetic changes of antifungal agents in adult patients receiving ECMO are limited to in vitro studies or case reports. While fluconazole does not seem to be affected by the ECMO circuit with a mean drug recovery of $91 \%$ at $24 \mathrm{~h}$, voriconazole concentrations appear to be significantly affected, with up to a $71 \%$ loss at $24 \mathrm{~h}[14,42]$. Despite adequate serum concentrations of caspofungin at recommended doses, data suggest some sequestration by the ECMO circuit [43]. Lipophilic formulations of amphotericin $\mathrm{B}$ as well as posaconazole and isavuconazole, both highly protein bound, may result in significant sequestration within the ECMO circuit.

When designing an appropriate antimicrobial dosing regimen for patients receiving ECMO, the biochemical 
properties of each drug should be considered, generally favoring a high initial concentration while monitoring for potential toxicities. Whenever possible, monitoring of drug concentrations, including peaks and troughs as appropriate, will help inform an effective dose and interval. Individualized dosing strategies may be necessary, especially when targeting a specific microorganism. Further insight into pharmacokinetic changes of antimicrobials in adult ECMO patients will be provided with the ongoing Analgesia, Sedation, and Antibiotic Pharmacokinetics during Extracorporeal Membrane Oxygenation (ASAP ECMO) trial [44].

\section{Limitations to current studies}

Although there are known pharmacokinetic changes occurring as a consequence of ECMO support, there are very limited data addressing the clinical outcomes associated with these observations. For example, agents used for sedation or blood pressure control can be titrated to predetermined clinical endpoints, permitting the bedside clinician to use a dose for which the effects may be easily observed and measured. However, in the absence of therapeutic drug monitoring, attainment of adequate antimicrobial concentrations may not be so readily observed, instead requiring the clinician to rely on surrogate endpoints, such as white blood cell counts or temperature curves, to assess effectiveness. Much of the existing data is limited to simulated circuits that do not account for metabolism or elimination. Additionally studies have not addressed how changing blood flow rates or using different ECMO configurations could impact the amount of drug sequestered within the circuit. Finally, many of the studies do not have control subjects, have not addressed the effects of circuits over longer time intervals, and have not studied the impact of additional extracorporeal circuits, such as continuous renal replacement therapy, on pharmacokinetic parameters.

\section{Future directions}

There is potential for novel strategies, such as altering the materials used in ECMO tubing or creating polymeric micelles for drug delivery, to minimize drug sequestration within the ECMO circuit. Absorption of lipophilic drugs to traditional medical grade PVC tubing containing di-ethylhexyl phthalate, a plasticizer, has been well established over the years. One in vitro study observed less adsorption capacity for lipophilic drugs when alternative materials, such as Teflon or silicone-caoutchouc mixture (SRT 460), were used [13]. Another method would be to encapsulate intravenous lipophilic drugs within micelles, a concept that has previously been demonstrated with gene therapy [45]. The idea is to solubilize appropriate portions of lipophilic drugs into the hydrophobic core, allowing attraction of the lipophilic tail to the surface of the ECMO tubing and drug release with minimal adherence to the surface of the tubing.

\section{Conclusion}

The pharmacokinetics and pharmacodynamics of drugs administered to critically ill patients are influenced by several factors, including the physiochemical properties of the drugs, the etiology and severity of the underlying illness, and the function of the organs responsible for drug metabolism. The presence of ECMO has an additional impact on drug distribution and metabolism, particularly due to increases in volume of distribution and sequestration by circuit components. Data are limited regarding the optimal regimen and dosing of sedatives and analgesics for critically ill patients receiving ECMO support, with the existing literature suggesting that, in many cases, higher amounts of analgosedation may be necessary to achieve therapeutic levels than would be expected for critically ill patients not receiving ECMO. Certain classes of antimicrobials may likewise be affected by $\mathrm{ECMO}$, potentially leading to sub-therapeutic drug concentrations if usual dosing regimens are used. Emerging data from the ASAP ECMO trial should help inform the appropriate administration of many commonly used antimicrobials, sedatives and analgesics in patients receiving ECMO.

\section{Acknowledgements \\ Not applicable.}

\section{Funding}

Publication costs were funded by the Division of Pulmonary, Allergy and Critical Care Medicine, Columbia University Medical Center.

\section{Availability of data and materials}

Not applicable.

\section{Authors' contributions}

$A D, D A$, and $D B$ contributed to the body of the manuscript. All authors read and approved the final manuscript.

\section{Competing interests}

DB is currently on the medical advisory boards of ALung Technologies and Kadence. All compensation for these activities is paid to Columbia University. ALD and DA declare that they have no competing interests.

Consent for publication

Not applicable.

Ethics approval and consent to participate Not applicable.

\section{Author details}

${ }^{1}$ Columbia University Medical Center, Department of Pharmacy, NewYork-Presbyterian Hospital, New York, NY 10032, USA. ²Division of Pulmonary, Columbia University College of Physicians and Surgeons, Allergy and Critical Care, 622 West 168th Street, New York, NY 10032, USA. 


\section{Published online: 21 March 2017}

\section{References}

1. Brodie D, Bacchetta M. Extracorporeal membrane oxygenation for ARDS in adults. N Engl J Med. 2011;365:1905-14.

2. Paden ML, Conrad SA, Rycus PT, Thiagarajan RR, Registry ELSO. Extracorporeal life support organization registry report 2012. Asaio J. 2012; 59:202-10.

3. Karagiannidis C, Brodie D, Strassmann S, et al. Extracorporeal membrane oxygenation: evolving epidemiology and mortality. Intensive Care Med. 2016:42:889-96.

4. Roberts JA, Lipman J. Pharmacokinetic issues for antibiotics in the critically ill patient. Crit Care Med. 2009:37:840-51.

5. Shekar K, Fraser JF, Smith MT, et al. Pharmacokinetic changes in patients receiving extracorporeal membrane oxygenation. J Crit Care. 2012;27:741.

6. Shekar K, Roberts JA, McDonald Cl, et al. Sequestration of drugs in the circuit may lead to therapeutic failure during extracorporeal membrane oxygenation. Crit Care. 2012;16:R194.

7. Preston TJ, Ratliff TM, Gomez D, et al. Modified surface coatings and their effect on drug adsorption within the extracorporeal life support circuit. J Extra Corpor Technol. 2010;42:199-202.

8. Preston $\mathrm{TJ}$, Hodge $\mathrm{AB}$, Riley JB, et al. In vitro drug adsorption and plasma free hemoglobin levels associated with hollow fiber oxygenators in the extracorporeal life support (ECLS) circuit. J Extra Corpor Technol. 2007;39:234-7

9. Rosen DA, Rosen KR, Silvasi DL. In vitro variability in fentanyl absorption by different membrane oxygenators. J Cardiothorac Anesth. 1990;4:332-5.

10. Wildschut ED, Ahsman MJ, Allegaert $K$, et al. Determinants of drug absorption in different ECMO circuits. Intensive Care Med. 2010;36:2109-16.

11. Mulla $\mathrm{H}$, Lawson $\mathrm{G}$, von Anrep $\mathrm{C}$, et al. In vitro evaluation of sedative drug losses during extracorporeal membrane oxygenation. Perfusion. 2000;15:21-6.

12. Bhatt-Meht $V$, Annich G. Sedative clearance during extracorporeal membrane oxygenation. Perfusion. 2005;20:309-15

13. Unger JK, Kuehlein G, Schroers A, Gerlach JC, Rossaint R. Adsorption of xenobiotics to plastic tubing incorporated into dynamic in vitro systems used in pharmacological research - limits and progress. Biomaterials. 2001;22:2031-7.

14. Shekar K, Roberts JA, Mcdonald Cl, et al. Protein-bound drugs are prone to sequestration in the extracorporeal membrane oxygenation circuit: results from an ex vivo study. Crit Care. 2015;19:164

15. Shekar K, Roberts JA, Barnett AG, et al. Can physicochemical properties of antimicrobials be used to predict their pharmacokinetics during extracorporeal membrane oxygenation? Illustrative data from ovine models. Crit Care. 2015;19:437.

16. Poole SK, Poole CF. Separation methods for estimating octanol-water partition coefficients. J Chromatogr B Analyt Technol Biomed Life Sci. 2003; 797:3-19.

17. Barr J, Fraser GL, Puntillo K, et al. Clinical practice guidelines for the management of pain, agitation, and delirium in adult patients in the ICU. Crit Care Med. 2013;41:263-306

18. Wagner D, Pasko D, Phillips K, et al. In vitro clearance of dexmedetomidine in extracorporeal membrane oxygenation. Perfusion. 2013;28:40-6.

19. Lemaitre $F$, Hasni $N$, Leprince $P$, et al. Propofol, midazolam, vancomycin and cyclosporine therapeutic drug monitoring in extracorporeal membrane oxygenation circuits primed with whole human blood. Crit Care. 2015;19:40

20. Shekar K, Roberts JA, Ghassabian S, et al. Sedation during extracorporeal membrane oxygenation - why more is less. Anaesth Intensive Care. 2012:40:1067-9.

21. Shekar K, Roberts JA, Mullany DV, et al. Increased sedation requirements in patients receiving extracorporeal membrane oxygenation for respiratory and cardiorespiratory failure. Anaesth Intensive Care. 2012;40:648-55.

22. DeGrado JR, Hohlfelder B, Ritchie BM, Anger KE, Reardon DP, Weinhouse GL. Evaluation of sedatives, analgesics, and neuromuscular blocking agents in adults receiving extracorporeal membrane oxygenation. J Crit Care. 2016;37:1-6

23. Der Nigoghossian C, Dzierba AL, Etheridge J, et al. Effect of extracorporeal membrane oxygenation use on sedative requirements in patients with severe acute respiratory distress syndrome. Pharmacotherapy. 2016;36:607-16
24. Tellor B, Shin N, Graeta TJ, Avidan MS. Ketamine infusion for patients receiving extracorporeal membrane oxygenation support: a case series. F1000Res. 2015;4:16.

25. Floroff CK, Hassig TB, Cochran JB, Mazur JE. High-dose sedation and analgesia during extracorporeal membrane oxygenation: A focus on the adjunctive use of ketamine. J Pain Palliat Care Pharmacother. 2016;30:36-40.

26. Dzierba AL, Brodie D, Bacchetta M, et al. Ketamine use in sedation management in patients receiving extracorporeal membrane oxygenation. Intensive Care Med. 2016:42:1822-3.

27. Buscher H, Vaidiyanathan S, Al-Soufi S, et al. Sedation practice in venovenous extracorporeal membrane oxygenation: an international survey. ASAIO J. 2013;59:636-41.

28. Dellinger RP, Levy MM, Rhodes A, et al. Surviving Sepsis Campaign: international guidelines for management of severe sepsis and septic shock: 2012. Crit Care Med. 2013:41:580-637.

29. Kumar A, Roberts D, Wood KE, et al. Duration of hypotension before initiation of effective antimicrobial therapy is the critical determinant of survival in human septic shock. Crit Care Med. 2006;34:1589-96.

30. Donadello K, Roberts JA, Cristallini S, et al. Vancomycin population pharmacokinetics during extracorporeal membrane oxygenation therapy: a matched cohort study. Crit Care. 2014;18:632.

31. De Rosa FG, Corcione $S$, Baietto $L$, et al. Pharmacokinetics of linezolid during extracorporeal membrane oxygenation. Int J Antimicrob Agents. 2013;41:590-1.

32. Gélisse $\mathrm{E}$, Neuville $\mathrm{M}$, de Montmollin $\mathrm{E}$, et al. Extracoporeal membrane oxygenation (ECMO) does not impact on amikacin pharmacokinetics: a case-control study. Intensive Care Med. 2016:42:946-8.

33. Shekar K, Roberts JA, Ghassabian S, et al. Altered antibiotic pharmacokinetics during extracorporeal membrane oxygenation: cause for concern? J Antimicrob Chemother. 2013;68:726-7.

34. Shekar K, Fraser JF, Taccone FS, et al. The combined effects of extracorporeal membrane oxygenation and renal replacement therapy on meropenem pharmacokinetics: a matched cohort study. Crit Care. 2014;18:565.

35. Donadello K, Antonucci E, Cristallini S, et al. Beta-lactam pharmacokinetics during extracorporeal membrane oxygenation therapy: A case-control study. Int J Antimicrob Agents. 2015;45:278-82.

36. Udy AA, Roberts JA, Boots RJ, Paterson DL, Lipman J. Augmented renal clearance: implications for antibacterial dosing in the critically ill. Clin Pharmacokinet. 2010;35:606-8.

37. Turner RB, Rouse S, Elbarbry F, et al. Azithromycin pharmacokinetics in adults with acute respiratory distress syndrome undergoing treatment with extracorporeal-membrane oxygenation. Ann Pharmacother. 2016;50:72-3.

38. Veinstein A, Debouverie $\mathrm{O}$, Grégoire $\mathrm{N}$, et al. Lack of effect of extracorporeal membrane oxygenation on tigecycline pharmacokinetics. J Antimicrob Chemother. 2012:67:1047-8.

39. Lemaitre F, Luyt CE, Roullet-Renoleau F, et al. Impact of extracorporeal membrane oxygenation and continuous venovenous hemodiafiltration on the pharmacokinetics of oseltamivir carboxylate in critically ill patients with pandemic (H1N1) influenza. Ther Drug Monit. 2012;34:171-5.

40. Mulla H, Peek GJ, Harvey C, et al. Oseltamivir pharmacokinetics in critically ill adults receiving extracorporeal membrane oxygenation support. Anesth Intensive Care. 2013;41:66-73.

41. Eyler RF, Heung M, Pleva M, et al. Pharmacokinetics of oseltamivir and oseltamivir carboxylate in critically ill patients receiving continuous venovenous hemodialysis and/or extracorporeal membrane oxygenation. Pharmacotherapy. 2012;32:1061-9.

42. Mehta NM, Halwick DR, Dodson BL, et al. Potential drug sequestration during extracorporeal membrane oxygenation: results from an ex vivo experiment. Intensive Care Med. 2007;33:1018-24.

43. Spriet I, Annaert P, Meersseman P, et al. Pharmacokinetics of caspofungin and voriconazole in critically ill patients during extracorporeal membrane oxygenation. J Antimicrob Chemother. 2009;63:767-70.

44. Shekar K, Roberts JA, Welch S, et al. ASAP ECMO: Antibiotic, sedative and analgesic pharmacokinetics during extracorporeal membrane oxygenation: a multi-centre study to optimise drug therapy during ECMO. BMC Anesthesiol. 2012:12:29.

45. Jones M, Leroux J. Polymeric micelles - a new generation of colloidal drug carriers. Eur J Pharm Biopharm. 1999;48:101-11. 